In S. Cotin and D.N. Metaxas, eds., "Proceedings of Medical Simulation:

International Symposium - ISMS 2004, Cambridge, MA, June 17-18, 2004,

Lecture Notes in Computer Science vol. 3078, Springer-Verlag, pp. 9-18.

\title{
The Effects of Testing Environment on the Viscoelastic Properties of Soft Tissues
}

\author{
Mark P. Ottensmeyer ${ }^{1}$, Amy E. Kerdok ${ }^{2,3}$, Robert D. Howe ${ }^{2,3}$, Steven L. Dawson ${ }^{1,4}$ \\ ${ }^{1}$ CIMIT Center for Surgical Simulation, \\ 65 Landsdowne St., ste. 142, Cambridge, MA, 02139, USA \\ ottensmeyer.mark@mgh.harvard.edu, sdawson@partners.org \\ http://www.medicalsim.org \\ ${ }^{2}$ Harvard University Division of Engineering and Applied Sciences, \\ Pierce Hall B11B/C, 29 Oxford St., Cambridge, MA 02138, USA \\ kerdok@fas.harvard.edu, howe@deas.harvard.edu \\ http://biorobotics.harvard.edu \\ ${ }^{3}$ Harvard/MIT Division of Health Sciences and Technology \\ ${ }^{4}$ Department of Radiology, Massachusetts General Hospital
}

\begin{abstract}
Mechanical properties of biological tissues are needed for accurate surgical simulation and diagnostic purposes. These properties change postmortem due to alterations in both the environmental and physical conditions of the tissue. Despite these known changes, the majority of existing data have been acquired ex vivo due to ease of testing. This study seeks to quantify the effects of testing conditions on the measurements obtained when testing the same tissue in the same locations with two different instruments over time. We will discuss measurements made with indentation probes on whole porcine livers in vivo, ex vivo with a perfusion system that maintains temperature, hydration, and physiologic pressure, ex vivo unperfused, and untreated excised lobes. The data show $>50 \%$ differences in steady state stiffness between tissues in vivo and unperfused, but only $17 \%$ differences between in vivo and perfused tests. Variations also exist in the time-domain and frequency domain responses between all test conditions.
\end{abstract}

\section{Introduction/Motivation:}

The mechanical properties of biological tissues are necessary for accurate surgical simulation and diagnostic purposes. Software-based simulation of surgical procedures relies on accurate representation of the mechanical response of tissues subject to surgical manipulations. If the tissue models or parameters are significantly different from reality, then negative training transfer may result from the use of the flawed simulator. Palpation, or manual evaluation of tissue mechanical response, has been used since the dawn of medicine, and numerous types of pathology cause changes in the viscoelastic character of tissue [1,2]. Should mechanical testing be used on this basis for diagnostic purposes, accurate measurements of healthy and diseased tissues are needed. 
Soft tissues not only have complex material properties that are difficult to characterize, but also exist in an environment that affects their intrinsic behavior. Testing the tissue in its natural state is ideal for ensuring accurate representations of the mechanical behavior we wish to characterize but difficult to achieve due to accessibility, ethical, variability, noise, and uncontrolled boundary condition issues. Despite these issues, several groups have recently developed instrumentation to measure tissue properties in vivo $[3,4,5,6,7,8]$. Although these groups have successfully made force-displacement measurements on tissues in vivo, the interpretation of these results remains to be understood.

The majority of existing data has been acquired under ex vivo conditions because this allows for precise control of boundary and loading conditions, provides access to appropriate testing sites and uses fewer animals $[9,10,11,12]$. However, testing soft tissues ex vivo drastically alters their properties and behavior [7, 13] and transplant researchers indicate that tissues lose their functional viability and structural integrity within hours $[14,15]$. A qualitative and quantitative understanding of the differences in measuring material properties from these different conditions is clearly needed.

We believe that to best understand the differences between testing conditions, measurements that can capture both the elastic and viscous properties of soft tissues need to be made on the same organ, at the same location, across various environmental conditions. Several researchers have made soft tissue measurements in various environmental states $[3,5,7]$ (in vivo, in situ, whole and partial organ ex vivo with and without controlling for hydration and temperature effects). Despite this, no examples were found in which tissues were tested, harvested and retested in the same location with the same instruments to examine the changes in tissue properties post mortem. Brown et al [7] have measured the first squeeze force-displacement and stress relaxation response of porcine liver using graspers across three different environmental conditions: in vivo, in situ, and ex vivo. However, their data reveal structural not material properties with complex boundary conditions. Since they only measured the first squeeze response there is no measure of repeatability within location or across condition.

This study seeks to quantify the effects of testing conditions on soft tissue material property measurements. We will discuss measurements made with two different indentation probes designed to capture the elastic and viscous material properties on whole porcine liver tissue in vivo, ex vivo with perfusion, ex vivo post-perfusion, and in vitro (i.e. warm ischemic partial organ tissue). These tests serve to verify the function of our perfusion system, examine the differences between in vivo, perfused and unperfused tissue, and to compare intact versus excised organ conditions.

\section{Methods}

We seek to quantify the responses of tissues under four different conditions, namely the in vivo case, the in vitro excised lobe case, and two different ex vivo whole organ cases including perfused testing, and testing on tissues that have been flushed of blood with the perfusate, but tested thereafter without being supported by the perfusion system. 
The following sections describe the perfusion system in detail, as well as the indentation testing apparatuses used to measure the viscoelastic response of the tissues under each test condition.

\subsection{Normothermic Extracorporeal Liver Perfusion System}

To accurately measure the mechanical properties of the liver, it is crucial that we maintain cellular integrity while keeping the organ in as natural a state as possible ex vivo. Thus we have built an apparatus similar in concept to normothermic extracoporeal perfusion systems using heparinzed Lactated Ringer's solution as the perfusate (see Fig. 1). The system stores this solution in reservoirs suspended at specified heights to obtain the appropriate physiologic pressures into the hepatic artery (100$120 \mathrm{mmHg}$ ) and portal vein $(15-20 \mathrm{mmHg})$. Both pressures and flow rates can be easily adjusted by altering the height of the reservoirs and by partially closing tubing clamps respectively. The perfusate is then allowed to drain via the intrahepatic vena cava into a bath where it is heated to a physiologic temperature (39C for pigs) and circulated to the reservoirs via a pump. The solution also flows over the organ to maintain hydration without having to submerge the organ. To ensure consistency in our measurements, the organ rests on a sturdy plate covered with fine grit sandpaper to localize and stabilize the area of tissue under study, and the perfusion pressure is held constant rather than mimicking physiologic pulsatile pressure.

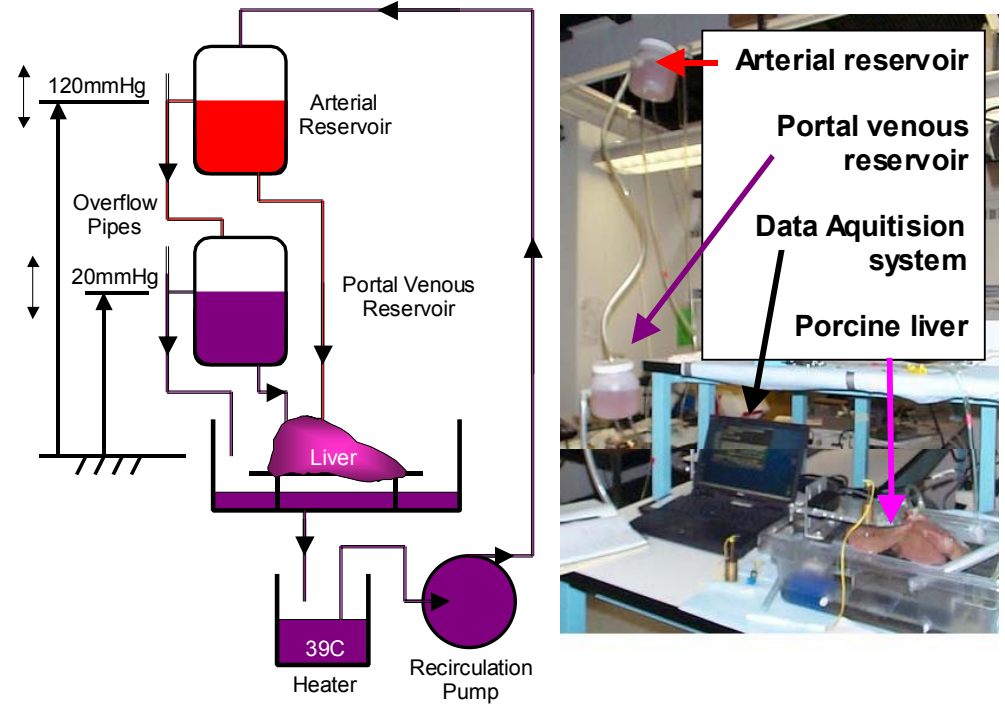

Fig. 1. (left) Our Normothermic Extracorporeal Liver Perfusion system schematic. (right) NELP system in use 


\subsection{Test Instruments}

We used two different indentation devices to measure the viscoelastic response of the tissue. The TeMPeST (Tissue Material Property Sampling Tool) allows us to measure the small strain frequency response of tissues, while the VESPI (Visco-Elastic Soft-tissue Property Indentation instrument) examines the large strain time-domain response (see Fig. 2).

TeMPeST 1-D. TeMPeST 1-D is a $12 \mathrm{~mm}$ diameter minimally invasive instrument, designed to measure the compliance of solid organ tissues within the linear regime. A $5 \mathrm{~mm}$ right circular punch vibrates the tissue while recording applied load and relative displacement. Mechanical bandwidth is approximately $80 \mathrm{~Hz}$ when in contact with organ tissues, however the force and position sampling frequency is up to $2 \mathrm{kHz}$, so measurements can be made to approximately $200 \mathrm{~Hz}$, depending on the material. Range of motion is $1 \mathrm{~mm}$ and forces up to $300 \mathrm{mN}$ can be exerted. It has previously measured the properties of porcine liver and spleen in vivo, rodent (rat) liver and kidney ex vivo $[6,16]$, and has been used in initial investigations of bovine, ovine and human vocal tissue samples ex vivo.

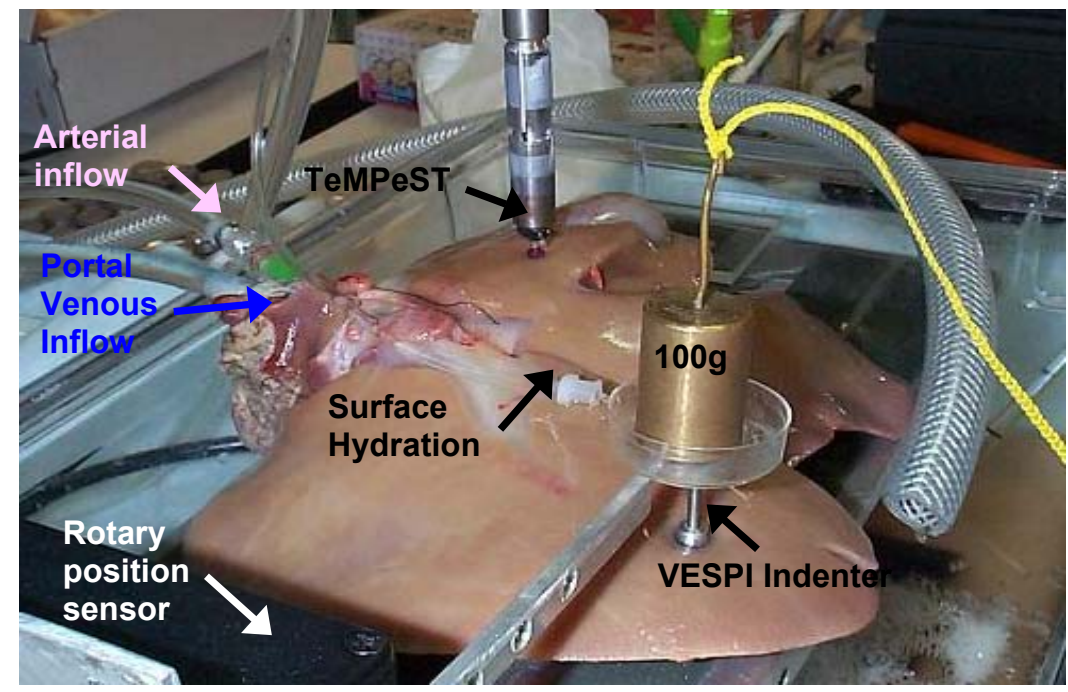

Fig. 2. The TeMPeST and the VESPI devices testing 2 separate locations on the same pig liver maintained in the normothermic extracorporeal perfusion system.

VESPI. The VESPI also performs normal indentation on tissues. It was designed for bench-top use, and subsequently modified to permit open surgical in vivo measurements as well. A $6 \mathrm{~mm}$ diameter flat punch rests, with only $3 \mathrm{~g}$ load due to counter weights, on the tissue surface until a standard laboratory mass is released (at zero velocity) onto a platform mounted co-axially with the indenter tip. The organ rests on the same platen to which the measurement arm and indenter tip are mounted. 
Loads from 20 to $100 \mathrm{~g}$ (nominal stresses of 6.2 to $31 \mathrm{kPa}$ ) generate much larger deformations than those created by the TeMPeST. Because of the large range of motion of the instrument, and the relative immobility of the organ resting on the platen (compared with TeMPeST testing), breathing does not need to be suspended during testing, enabling much longer data acquisition periods (typically 300 seconds). During this period, the angular position of the measurement arm is measured at a rate of $1 \mathrm{kHz}$ using a miniature contactless rotary position sensor (Midori America Corporation, CA) (resolution $13.7 \mu \mathrm{m}$, signal to noise $\sim 100: 1$ ). Since the lever arm has a length of $11.5 \mathrm{~cm}$, and the maximum depth of indentation was on the order of $10 \mathrm{~mm}$, small angle approximation was assumed and thus the voltage from the rotary sensor is converted directly to indentation depth using a linear gain.

\subsection{Test Protocol}

In vivo tests were performed on deeply anesthetized animals on assisted ventilation with $100 \%$ oxygen. The TeMPeST instrument was used to acquire compliance data on either one or two locations on the liver during data acquisition periods of approximately 20 seconds. During this time, ventilation was suspended to prevent pulmonary motions from saturating the position sensor measurements. Indentations using the VESPI device were made at the same location(s), but without the necessity for suspending ventilation. Organ thickness measurements were taken prior to every VESPI measurement with a $0-25 \mathrm{~mm}$ dial indicator. Initial position senor values were noted and loads were applied for 300 seconds. Once the load was removed the organ was allowed to recover to its preindented state (typically 200s, as was determined by comparing the current position to the preloaded value).

Following in vivo testing, heparin was injected systemically to prevent clotting, and the animal was sacrificed. The liver was harvested, and a lobe was removed and tested immediately with the TeMPeST (in vitro testing). The cut surface of the reminder of the organ was cauterized to prevent leakage and the organ was flushed with heparinized lactated Ringer's solution, packed in ice and transported to the laboratory.

Upon arrival (60-80min post-sacrifice), the liver was connected to the arterial and hepatic venous perfusate reservoirs, and allowed to come to physiological temperature and pressure before testing was resumed. TeMPeST and VESPI tests were performed in the same locations as the in vivo tests to minimize variation in the measurements due to the unknown locations of large vessels or connective tissues within the organ. Testing on the excised (untreated) lobe was also performed over time with both instruments. Times of sacrifice, and initiation and termination of perfusion were recorded to permit examination of measurements over time.

Following the completion of testing on the perfused organ (typically 2 hours), perfusate flow was stopped, and the organ was tested again over time to observe any further changes in the response (typically 1 hour).

The research was conducted in compliance with the Animal Welfare Act Regulations and other Federal statutes relating to animals and experiments involving animals and adheres to the principles set forth in the Guide for Care and Use of Laboratory Animals, National Research Council, 1996. 


\section{Results}

Variations in the measured responses are observed between all of the conditions under consideration, including both changes in the measured tissue stiffness and the time dependent viscous character of the responses. The measurements performed with the TeMPeST and VESPI will be shown in the following sections.

\subsection{TeMPeST Results}

Fig. 3 shows the frequency response calculated from the ratio of the FFTs of the position and force signals, together with the ideal first order filter response of a Voigt tissue model. As the response appears to have a $-20 \mathrm{~dB} /$ decade slope after the break, and the phase lag at high frequencies is approximately $-90^{\circ}$, the Voigt/first order response is a reasonable first approximation to fit the results. It is observed that the in vivo measurements show the highest compliance (lowest stiffness), while the perfused tissues are stiffer, and the unperfused tissues (after prior perfusion) are the stiffest. In addition, the break of the response shifts to higher frequencies in each of these cases.
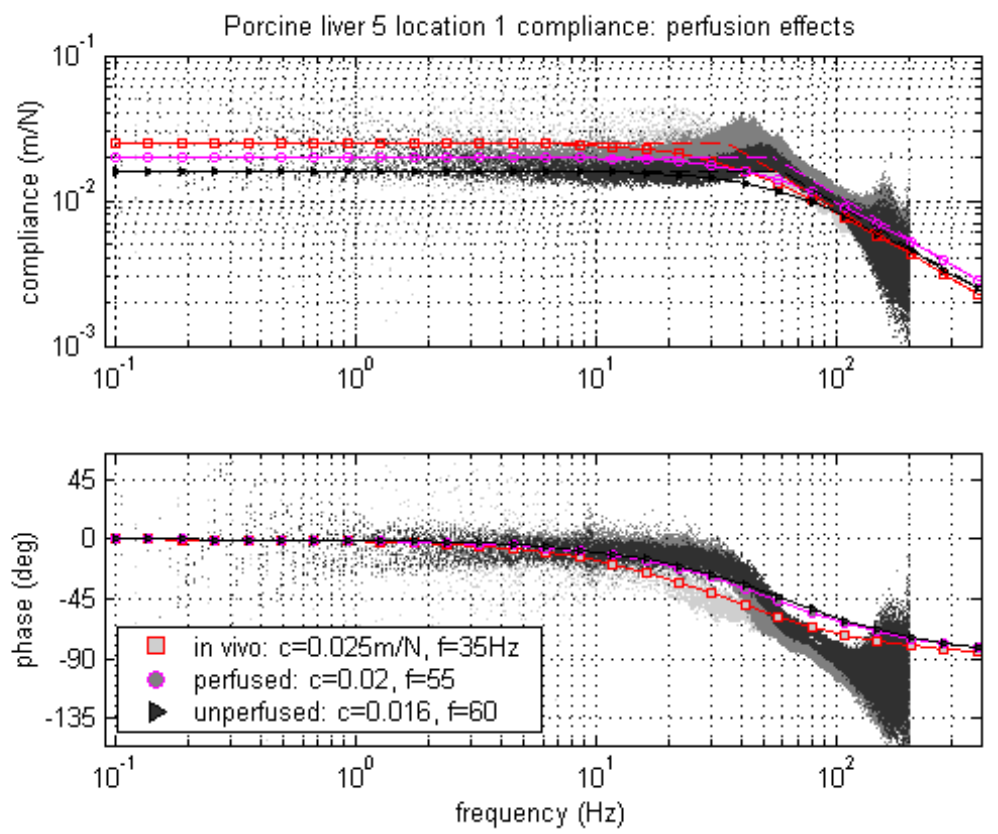

Fig. 3. Frequency response of tissue measured with TeMPeST and Voigt model approximation of tissue response. First order filter characteristics include asymptotes to better show characteristic frequency (dashed lines). 


\subsection{VESPI Results}

Fig. 4 shows representative results for three conditions made on the same liver taken at the same location. Strain is calculated as depth of indentation normalized with respect to thickness measured prior to that specific test. It can be seen that the tissue in vivo is softer than perfused tissue, which in turn is softer than the unperfused organ. Not shown is the data from the in vitro experiment, which was much softer than all other conditions and which never fully achieved a steady strain state. Most significantly, the perfused steady state strain is within $17 \%$ of the in vivo value, much closer than the unperfused strain, which differs by more than $50 \%$ (considering $3^{\text {rd }}$ indentation for each case).

We were also interested in examining the repeatability of the measurements within location. Fig. 4 shows that after allowing the tissue to recover fully after testing, the in vivo time responses are very similar to each other, as are the perfused tests. The unperfused test, because an external source of pressurized perfusate is no longer available, does not recover, and significant changes are observed between the first and third measurements.
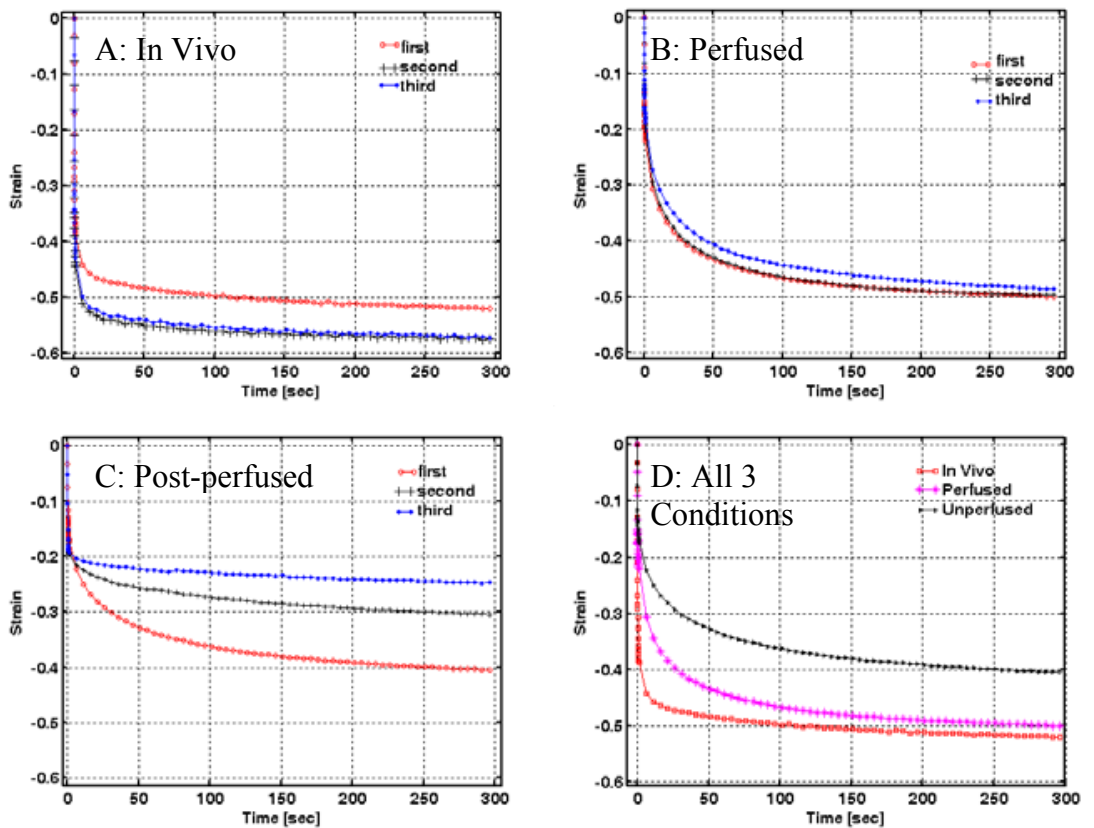

Fig. 4. VESPI response for three of the conditions under consideration on a $27 \mathrm{~kg}$ pig liver at the same location. (A) In vivo $100 \mathrm{~g}$ load. The second and third indentations were taken 10 and 50 minutes after the first. (B) Perfused $100 \mathrm{~g}$ load. The second and third indentations were taken 20 and 48 minutes after the first. (C) Unperfused $100 \mathrm{~g}$ load. The first indentation was taken 1 minute post perfusion while the second and third were 12 and 25 minutes after that. (D) Results of the first indentation performed at each condition. 
Lastly, the curvature of the results provides insight to the creep time constants in the tissue. A quantitative analysis of the results will need to be performed, but qualitatively it can be seen that the curves of the various conditions are indeed different suggesting that the testing conditions alter the viscous properties of the tissue.

\section{Conclusions and Future Work}

Humans have the ability to distinguish between seven different levels of unidimensional stimuli [17]. Despite this rather coarse just noticeable difference, it has also been shown that haptically humans can feel differences in force as small as $10 \%$ [18]. For this reason, employing measurements from unperfused tissue as a proxy for perfused (or in vivo) data may result in significant inaccuracies in surgical simulators for training. For example, if one becomes accustomed to manipulating virtual tissues that are stiffer than real ones, excessive, possibly damaging forces may be applied when the trainee reaches the operating room. We hypothesized that in creating an environment that closely approximates in vivo conditions, we can maintain the mechanical viability of the tissue such that the properties we measure ex vivo are comparable to the in vivo properties and that without such an environment, the material properties are altered far beyond $10 \%$.

Each instrument shows variations in the stiffness and damping properties of the liver depending on the test condition. However, the large deformation time responses, when using the perfusion apparatus, approach those of tissues tested in vivo. The pressurized flow of perfusate permits the organ to fully recover after testing, just as the flow of blood through the living organ would. In addition the static pressure applied to the perfusate provides an internal "boundary condition" to the tissue which is present (at least on average) in the living organ, but is absent when testing tissues on the lab bench, even if immersed in physiological solution. Lastly, testing whole organs versus cut specimen provides a more accurate reference state containing residual stresses and strains.

The TeMPeST measurements show the high frequency response of the tissue, showing a slight increase in the break frequency after the tissues have been perfused. One possible explanation is that the Lactated Ringer's solution, mostly water, behaves as a Newtonian fluid, with a viscosity lower than that of the non-Newtonian blood that normally perfuses the tissues. Whether this is a noticeable error from the perspective of simulation is a question that cannot be answered in this study. If diagnostic applications are envisioned, it may be necessary to refine the perfusion system further to ensure a closer match in behavior over a wide range of time scales.

More recent examination of the literature as well as input from a pathologist examining some samples taken of the liver over time in each condition have shown that the perfusion pressure for the hepatic portal branch of the system is currently too high. In particular, the $20 \mathrm{mmHg}$ value is more than double the published value of $9 \mathrm{mmHg}$ [19]. This over pressure is most likely the reason why the shape of the VESPI creep curves between the in vivo and ex vivo perfused states were different. The increased pressure probably also accounted for some of the cellular dissociation seen histologically. Measurements of in vivo portal venous and arterial pressure will be performed 
in future experiments and the static pressures of the system will be altered accordingly. Osmotic and oncotic pressure issues may also be playing a role. Discussions with transplant surgeons are underway to better improve our perfusate recipe for future tests.

Tests scheduled, but not yet performed include testing whole organs over time in both the untreated (directly harvested) case and in the flushed-unperfused case. Also, a test will be conducted using the VESPI to determine the amount of mechanical damage (from analyzing histological samples) on the organ after the first load as a function of load.

In conclusion, it was confirmed that untreated tissues behave much differently than tissues in vivo, and that the perfusion system provides a suitable environment for testing whole organ tissues. The maintenance of hydration, temperature, osmotic balance and internal pressure are necessary to permit extended testing of whole organs on the lab bench, where testing can be conducted more conveniently than in the operating room. In addition, the perfusion system will enable the use of organs harvested from other sources, without ever performing dedicated in vivo tests, reducing the cost of testing and the ethical and administrative issues of in vivo testing. It is recommended that researchers performing soft tissue testing on solid organs make use of similar systems to provide mechanical function support to tissues tested in the laboratory setting.

\section{Acknowledgements}

Support for this work has been provided by a grant from the US Army, under contract number DAMD 17-01-1-0677. The ideas and opinions presented in this paper represent the views of the authors and do not, necessarily, represent the views of the Department of Defense.

\section{References}

1. P.S. Wellman. Tactile Imaging. Ph.D. Thesis, Division of Engineering and Applied Sciences, Harvard University, Cambridge (1999) p137.

2. T. A. Krouskop, T. M. Wheeler, F. Kallel, B. S. Garra, T. Hall. Elastic Moduli of Breast and Prostate Tissues Under Compression. Ultrasonic Imaging, 20 (1998) pp260-274.

3. I. Brouwer, J. Ustin, L. Bentley, A. Sherman, N. Dhruv, F. Tendick. Measuring In Vivo Animal Soft Tissue Properties for Haptic Modeling in Surgical Simulation. Proc. Medicine Meets Virtual Reality 2001, Newport Beach, CA. IOS Press. pp69-74.

4. J. Kim, B.K. Tay, N. Stylopoulos, D.W. Rattner, M.A. Srinivasan. Characterization of Intra-abdominal Tissues from In Vivo Animal Experiments for Surgical Simulation. Proc. Medical Image Computing \& Computer Assisted Intervention (MICCAI), Montreal, Canada, (2003) pp206-213.

5. F.J. Carter, T.G. Frank, P.J. Davies, D. McLean, A. Cuschieri. Measurements and modelling of the compliance of human and porcine organs. Medical Image Analysis, 5(4) (2001) pp231-6. 
6. M.P. Ottensmeyer. In vivo measurement of solid organ visco-elastic properties. Proc. Medicine Meets Virtual Reality 02/10 (MMVR02/10), Newport Beach, CA. IOS Press. (2002) pp328-33.

7. J. D. Brown, J. Rosen, Y. S. Kim, L. Chang, M. Sinanan, B. Hannaford. In-Vivo and InSitu Compressive Properties of Porcine Abdominal Soft Tissues. Proc. Medicine Meets Virtual Reality 11, Newport Beach, CA. IOS Press. (2003) pp26-32.

8. K. Miller, K. Chinzei, G. Orssengo, P. Bednarz. Mechanical properties of brain tissue invivo: experiment and computer simulation. J. Biomechanics, 33 (2000) pp1369-1376.

9. K. Miller. Biomechanics of Soft Tissues. Medical Science Monitor, 6 (2000) pp158-167.

10.M. Farshad, M. Barbezat, P. Flueler, F. Schmidlin, P. Graber, P. Niederer. Material Characterization of the Pig Kidney in Relation with the Biomechanical Analysis of Renal Trauma. Journal of Biomechanics, 32 (1999) pp417-425.

11.M. Kauer, V. Vuskovic, J. Dual, G. Szekely, M. Bajka. Inverse Finite Element Characterization of Soft Tissues. Proc. Medical Image Computing and Computer-Assisted Intervention, (MICCAI) Utrecht, Netherlands (2001) 128-136.

12.T. Hu, J. Desai. A Biomechanical Model of the Liver for Reality-Based Haptic Feedback. Proc. Medical Image Computing \& Computer Assisted Intervention (MICCAI), Montreal, Canada, (2003) pp75-82.

13.Y. C. Fung, Biomechanics: Mechanical Properties of Living Tissues, second ed. New York: Springer-Verlag, 1993.

14.M. Schon, O. Kollmar, S. Wolf, H. Schrem, M. Matthes, N. Akkoc, N. Schnoy, P. Neuhaus. Liver Transplantation After Organ Preservation with Normothermic Extracorporeal Perfusion. Annals of Surgery, 233 (2001) pp114-123.

15.K. P. Platz, A. R. Mueller, C. Schafer, S. Jahns, O. Guckelberger, P. Nehaus. Influence of Warm Ischemia Time on Graft Function in Human Liver Transplantation. Transplantation Proceedings, 29 (1997) pp3458-3459.

16.C. Bruyns, M.P. Ottensmeyer. Measurements of Soft-Tissue Mechanical Properties to Support Development of a Physically Based Vrtual Animal Model. Proc. Medical Image Computing and Computer-Assisted Intervention, MICCAI 2002, Tokyo, Japan (25-28 Sept 2002) pp282-289

17.G. Miller. The magic number seven plus or minus 2: some limits on our capacity for processing information. The Physiological Review, 63 (1955) pp 83-97.

18.S. Allin, Y. Matsuoka, R. Klatzky. Measuring Just Noticeable Differences for Haptic Force Feedback: Implications for Rehabilitation. Proc. 10th Symposium On Haptic Interfaces for Virtual Environments \& Teleoperator Systems (HAPTICS '02), Los Alamitos, CA, IEEE. pp299-302.

19.A. Rasmussen, C. Skak, M. Kristensen, P. Ott, P. Kirkegaard, N.H. Secher. Preserved arterial flow secures hepatic oxygenation during haemorrhage in the pig. Journal of Physiology, 516(2) (1999) pp539-548. 